4 | 2017

Technologies numériques et diffusion de l'information pendant les périodes de conflits et de crise dans le monde

\title{
Diversité de sources d'information et processus décisionnel en période de crise
}

Diversity of information source and decision process in time of crisis

Informations quellen vielfalt und entscheidungsprozesse in der krise

Fleur Nadine Ndjock

\section{OpenEdition}

\section{Journals}

Édition électronique

URL : http://journals.openedition.org/ctd/859

DOI : $10.4000 /$ ctd. 859

ISSN : 2491-1437

Éditeur

Chaire Unesco Pratiques émergentes en technologies et communication pour le développement

Référence électronique

Fleur Nadine Ndjock, « Diversité de sources d'information et processus décisionnel en période de crise ", Communication, technologies et développement [En ligne], 4 | 2017, mis en ligne le 04 septembre 2017, consulté le 29 mars 2021. URL : http://journals.openedition.org/ctd/859 ; DOl : https://doi.org/ $10.4000 /$ ctd. 859

Ce document a été généré automatiquement le 29 mars 2021.

Communication, technologies et développement 


\title{
Diversité de sources d'information et processus décisionnel en période de crise
}

\author{
Diversity of information source and decision process in time of crisis \\ Informations quellen vielfalt und entscheidungsprozesse in der krise
}

\author{
Fleur Nadine Ndjock
}

\section{Introduction}

Une source d'information est un document, livre, personne, un outil ou autre matériel à partir duquel vous pouvez obtenir de l'information liée à un fait ou un événement. C'est l'origine, la provenance, la naissance d'une information. Parler de source d'information c'est évoquer la manière dont l'information s'est fait connaître au public. De nos jours, avec le boom technologique, les sources d'information sont de formes très variées. On distingue des sources dites "classiques» ou "traditionnelles»: télévision, presse, radio et les nouvelles sources d'information liées à l'évolution d'internet: ce sont principalement les sites web et les réseaux sociaux. Le choix de la source dépend de plusieurs critères entre autres: l'accessibilité, la mise à jour, la pertinence et la fiabilité. Ces deux derniers critères sont cruciaux surtout en temps de crise ou de conflit, car aboutissant à la prise de décision. Or, la multiplication des sources d'information s'accompagne aussi de notables changements dans la production et la diffusion de l'information. Il devient alors nécessaire de s'interroger sur les enjeux de la communication en temps de crise et l'impact de la diversité des sources d'information dans le processus décisionnel. Nous définissons ce dernier comme étant un enchaînement ordonné d'opérations cognitives répondant à un certain schéma allant de l'identification d'un problème à la compréhension de l'environnement, la vérification des sources pertinentes d'information, à la collecte de données, à l'interprétation des indicateurs et aboutissant à la prise de décision. À notre avis, en période de crise, il est étroitement lié à la disponibilité et à la pertinence de 
l'information. En effet, en temps de crise, la communication a pour rôle d'informer, de dissuader et de permettre de prendre des décisions. Par exemple, savoir s'il faut se mettre à l'abri ou non, connaître la position de «l'ennemi », savoir ce que ci se passe, connaitre l'ampleur de la crise, etc. Toutes ces informations collectées permettront de prendre la «bonne » décision. Celle-ci est considérée comme un «processus cognitif complexe visant à la sélection d'un type d'action parmi différentes alternatives " (Barbalat, 2007: 91-105) et nous savons qu'en temps normal et particulièrement en période de crise, prendre une décision est un acte crucial d'autant plus que la décision doit être rapide et efficace. Pour cette raison, le décideur doit disposer de l'information pertinente. Or, il se trouve que plusieurs facteurs influencent la communication de l'information en temps de crise ou de conflit. Ces facteurs sont déterminants dans la demande et le traitement de l'information et jouent un rôle primordial dans la prise de décision finale. L'environnement conflictuel, la diversité des sources d'information, le problème de crédibilité des médias rendent particulièrement ardus la détermination des indicateurs, la collecte et le traitement de l'information. Le défi pour celui qui doit décider est d'en prendre conscience et d'avoir une capacité d'adaptation au problème auquel il fait face. Nous pensons que pour y arriver, il est important d'appliquer une méthodologie rigoureuse et efficace de l'ensemble du processus qui commence dès l'accès à l'information jusqu'à la prise de décision. Notre travail se divise en trois parties : après la spécification de la finalité de la communication dans un contexte de conflit ou de crise, nous essayerons d'identifier les problématiques liées à la diversité des sources d'information et leur impact dans le processus de prise de décision et enfin nous proposerons une méthodologie qui guidera le décideur dans la collecte et le traitement de l'information en période de crise.

\section{La finalité de la communication dans un contexte de conflit ou de crise}

2 Il faut entendre par communication, le partage, la transmission à quelqu'un de quelque chose, d'un sentiment, d'un état, d'une information ... De nombreux chercheurs ont donné leur approche définitionnelle de la communication, au nombre desquels Henriquez, E (1971). Cité par Cazeneuve: c'est le processus par lequel une source d'informations $\mathbf{A}$ tend à agir sur un récepteur d'informations $\mathbf{B}$ de manière à provoquer chez celui-ci l'apparition d'actes ou de sentiments permettant une régulation des activités de $\mathbf{B}$ ou du groupe auquel appartiennent A et B (Cazeneuve, 1963 : 11-17). Pour Akin Saliou, la science de la communication a pour objet justement, la conceptualisation et la rationalisation de tout ce processus de transmission de l'information entre deux entités, qui peuvent être des individus, des groupes d'individus voire même des machines (Akin, $2008: 16$ ). Nous pouvons ici faire le lien avec notre sujet. En effet, ces auteurs nous apprennent qu'en situation de crise, le rôle du décideur est de diffuser l'information nécessaire de manière à rassurer son environnement interne et externe.

3 Traditionnellement, la chaîne de transmission de l'information comprend :

- un émetteur qu'on appelle aussi expéditeur ;

- un récepteur qu'on appelle également destinataire ;

- un message constitué d'un ensemble de signes transmis de l'émetteur au destinataire ;

- un code qui doit être compréhensible et connu par les deux parties, par exemple la langue de communication qui sert à transmettre et à comprendre le message ; 
- un canal de transmission qui est l'ensemble des moyens par lesquels une information est transmise ;

- un contexte représentant les circonstances dans lesquelles s'insère la communication. Le contexte est déterminant dans la compréhension du message par le récepteur. Car selon celui- ci, un même message peut être perçu d'une manière différente. Par exemple, en temps de conflit ou d'attaque, le retentissement de la cloche ou une sirène peut signifier : « courir à l'abri le plus proche » et la même cloche en temps de paix peut simplement rappeler quelle heure il est. »

4 Toute société est susceptible de connaître un jour une situation dite de crise ou passer par un temps de conflit. La communication devient alors un élément majeur de sa gestion, de sa résolution, mais plus encore de sa sortie. Même si la gestion de chaque crise est unique, communiquer par ces temps concourt vers le même objectif ainsi que le suggère (Courtot, 2001); permettre de :

- fournir une meilleure connaissance de sa situation et de son niveau d'exposition aux risques; conduire à une meilleure maîtrise du conflit ou de la crise, en s'ajustant aux diverses évolutions de son environnement et en organisant la réactivité face aux divers événements susceptibles de se produire et de modifier son déroulement ;

- faciliter la prise de décision en fonction de l'urgence de la situation et de la faisabilité des actions de maîtrise, d'obtenir une meilleure définition du niveau de priorité des actions à engager, et de concevoir le meilleur compromis possible ; accroître les chances de succès du projet, grâce à une meilleure compréhension et identification des risques encourus, et une meilleure définition des actions à entreprendre pour s'en prémunir.

5 Toutefois, comme le souligne Courtot, il convient de rappeler que le rôle de la communication ne se réduit pas, comme le soulignent (Joly \& Muller, 1994) à une simple transmission d'informations ou à un échange de données sur les événements d'actualité et leurs conséquences. C'est également, la rencontre de personnes autour d'un sujet sur lequel chacun peut et doit s'exprimer. Parce que la communication, argumente-t-il, facilite, d'une part, la mise en commun de connaissances, le partage de savoirs et d'expériences, contribuant ainsi à une augmentation des discussions et des confrontations d'idées et de points de vue. D'autre part, elle permet, grâce aux nombreuses sources, d'obtenir de chacun, une prise de conscience partagée des risques encourus, un dialogue approfondi sur leurs causes et leurs conséquences, un partage d'informations pertinentes pour être a minima sensibilisés aux problèmes rencontrés, contribuant ainsi à créer le climat de confiance et de coopération.

6 La prise de décision serait donc liée, sans aucun doute, à la collecte d'informations née $\mathrm{du}$ dialogue entre les acteurs engagés dans la situation de crise. Ceci est d'autant plus vrai qu'en période de crise toutes les sphères de l'organisation sont avides d'information. Même s'il est de la responsabilité du décideur de prendre une décision, il est de son devoir de favoriser une communication participative de manière à susciter des points de vue divergents permettant à celui-ci de prendre une décision qui serait la résultante d'une mutualisation des idées. 


\section{Les problématiques liées à la diversité des sources d'information}

7 La vision mécanique de la communication est, de nos jours, désuète. En effet, il y a une vingtaine d'années, la communication de crise était essentiellement faite à coup de communiqués de presse, de prises de parole, de messages clés, et de passages savamment orchestrés dans les médias. De nos jours, la pluralité des conflits, des acteurs, la diversification des sources d'information notamment le développement d'internet et l'arrivée des réseaux sociaux font surgir des problématiques jusqu'ici inconnues. On peut noter entre autres :

\section{Du public-spectateur au public-acteur}

8 Selon (Libaert, 2011 : 9-15), «Plus que jamais, le public participe de la communication. De passif, il devient non seulement relais, mais également acteur-sensible, jusqu'à peser - parfois lourdement - dans le contrat communicationnel ». Constamment en mutation, le monde de la communication change en mêmetempsqu'ilsecomplexifie. Lepublicparticipedeplusenplusàlaconstructiondelacommunication à une échelle égale à celle des médias modifiant irrévocablement la relation entre émetteur / récepteur. Aujourd'hui le récepteur se rebiffe et décrypte la communication, analyse et participe à sa construction.

9 Avec le développement d'internet, certains professionnels constatent même des modifications de la structure de la communication dans ses aspects contractuel et transactionnel. Libaert (2011) relève les mutations suivantes :

- le lieu : une translation du lieu des médias traditionnels vers les réseaux sociaux;

- la dé-hiérarchisation : chaque individu, chaque groupe social veut faire entendre sa voix à égale hauteur des médias ;

- la réactivité : chaque stimulation provoque des réactions immédiates et une contagion de l'émotion à une vitesse et une échelle inégalées ;

- la fin de la toute-puissance médiatique : la presse se trouve confrontée au spectateur qui commente en direct, non seulement les événements, mais les choix éditoriaux et de réalisation, lorsqu'elle n'est pas concurrencée par les réseaux sociaux ;

10 Houllier (2009) considère que les enjeux de la communication ont changé si l'on s'intéresse au risque qu'encourent les médias d'être dépossédés de leur capacité stratégique au profit d'une sensibilité accrue aux stimuli et en incapacité de garder un cap stratégique dans leur communication. Plus que jamais, le public a un impact sur l'agenda, définit les codes et surtout peut avoir des effets notoires par son effet de masse.

\section{Effets de loupe et de halo}

11 L'effet de loupe peut être défini comme étant le fait de donner une importance démesurée à certains faits. Couture (2005: 55) adhère à ce propos quand il définit l'effet de loupe comme étant le fait de "partir d'une situation précise, d'un cas (personnel ou non), d'exemples, de petits faits et de construire une histoire », car d'après lui, les journalistes 
savent que le public n'aime pas les grandes théories. La loupe va ainsi proposer une histoire qui va donner des impressions au bout du compte.

D'après une étude réalisée en Belgique en faveur d'une journée d'études ${ }^{1}$ sur le " sentiment d'insécurité et impact des médias " (Goor, 2008 :9-16), la question des médias et du sentiment d'insécurité apparaît de façon transversale dans la plupart des résultats. Ceux-ci font apparaître que les participants dans leur grande majorité sont d'un commun accord à propos de l'influence des médias sur leur sentiment d'insécurité. Ils mettent en cause la logique de fonctionnement des médias qui les conduit à se focaliser sur ce qui ne va pas comme le confirme ce témoignage :

«Une bonne nouvelle n'est pas une nouvelle, mais une mauvaise nouvelle est toujours une nouvelle pour les médias. On aime bien raconter des choses affreuses et dangereuses, sinon, ça n'intéresse personne. On en parle trop, mais enfin, ça fait vivre les médias » (Goor, $2008: 11)$.

Il arrive que les médias fassent l'amalgame aux yeux du public qu'il induit en erreur et conduit à un sentiment de frustration pour les concernés. L'exemple d'un témoignage personnel en France qui illustre au mieux l'effet de loupe sur la situation de mon pays : «à l'instant où je dis que je suis camerounaise, les visages se ferment et les gens me prennent subitement en pitié, ils me demandent comment réussissons-nous à vivre avec la guerre au Cameroun? J'essaie de leur expliquer qu'il n'y a pas de guerre au Cameroun, mais personne ne m'écoute parce que les médias véhiculent une autre image des attaques sporadiques du groupe Boko-Haram dans les villages au nord du pays ».

14 Toujours d'après les résultats de l'étude belge ${ }^{2}$ :

« la plupart des journalistes belges reconnaissent explicitement que les journalistes ont une responsabilité claire, mais estiment que cette responsabilité n'implique pas une culpabilité des médias: ils ne sont qu'un des acteurs dans une société très complexe et ne sont donc certainement pas les seuls en cause. Les médias sont un miroir de la société. »

A l'inverse, la distorsion orientée et le rejet est le résultat de l'effet de halo encore appelé effet de contamination qui est la tendance à généraliser une première impression qu'on a de quelqu'un ou d'un phénomène. Mis en évidence de manière empirique par Thorndike (1922) et démontrée par Asch (1990), les spécialistes s'accordent à reconnaître l'influence des médias par l'effet de halo. Une caractéristique jugée négative à propos d'une personne ou d'une collectivité a tendance à rendre plus négatives les autres caractéristiques de cette personne, même sans les connaître (et inversement pour une caractéristique positive). C'est ainsi qu'au cœur de la crise d'Ebola en Afrique de l'Ouest entre juillet et août 2014, alors que les États concernés et le Nigéria en particulier mettaient tout en œuvre pour enrayer la maladie, les médias par les nouvelles et les images diffusées créaient la psychose dans le monde entier. Seuls les plus téméraires pouvaient prendre le risque de voyager pour découvrir une fois sur place (sans ignorer le danger) que l'horreur et le risque n'étaient pas aussi omniprésents que les présentaient les médias et les dispositions sont prises pour limiter les dégâts. Mon expérience personnelle en est un exemple concret. Les compagnies aériennes ont suspendu les vols pour et en provenance du Nigéria cependant, je devais m'y rendre pour une conférence à Lagos en dépit de la dissuasion des médias : "Transition from Observation to Knowledge to Intelligence (TOKI)» du 20-22 août 2014. À l'aéroport, ma surprise fut grande de constater le contraste entre ce que rapportaient les médias et la réalité. Certes, des mesures de sécurité avaient été prises à 
la hauteur de la catastrophe, mais rien à voir avec ce que présageaient les médias. Cependant, alors que les participants étrangers sont arrivés au colloque, ceux des villes du Nigéria comme Ibadan à $120 \mathrm{~km}$ ou Abuja un peu plus loin à $800 \mathrm{~km}$, n'ont pu faire le déplacement à cause des médias.

Toutefois, à la lecture de ce qui est dit jusqu'ici, il serait péremptoire et dangereux de penser que les médias jouissent uniquement d'une influence négative en période de crise ou de conflit. C'est l'occasion pour nous de nuancer nos propos tout en relevant que la diversité de sources permet de croiser l'information, de la comparer et d'établir leur pertinence. En période de crise ou de conflit, celle-ci joue son rôle premier qui est d'informer le public. Ce rôle est prépondérant pour la sécurité, qu'elle soit individuelle ou collective, physique ou psychologique, économique ou politique. L'information éveille les consciences et à plusieurs reprises, les médias ont joué ce rôle d'éveil des consciences que ce soit par images interposées ou de posts sur les réseaux sociaux. Prenons quelques exemples de cet éveil renforcé par la digitalisation du principe du bouche- à-oreille : la mobilisation mondiale autour des problèmes écologiques de notre planète sur le réchauffement climatique, les appels au tri ainsi qu'au recyclage, la mobilisation mondiale autour des questions des migrants, l'appel à témoins qui a abouti à l'identification des auteurs du double attentat de Boston en 2013.

\section{Course à l'audience}

17 Paradoxalement, la recherche du sensationnel dans les médias, et notamment ceux qui diffusent l'information en continu dénote une incompréhension certaine des enjeux relatifs à la communication en temps de conflit ou de crise. Souvent, les journalistes (en plateau) comblent les temps morts par des témoignages et des expertises. Ce traitement médiatique qui répond à la fois à la curiosité des spectateurs, aux exigences du direct ainsi qu'à la course à l'audience conduit à la recherche d'informations par tous les moyens. Il arrive que les informations soient diffusées sans traitement préalable ce qui donne l'impression de contradiction et de balbutiement. Le cas des attentats récents de Belgique en est une preuve concrète. Le nombre de morts et de blessés n'a cessé de fluctuer au gré du temps et des médias à cause de l'empressement de journalistes et experts à dévoiler les détails glanés ici et là dans une course à l'audience toujours impitoyable.

\section{II n'y a pas de communication (information) neutre}

18 Aucune communication n'est gratuite. «Le livre véhicule une culture, des valeurs, son apparence suscite des réactions affectives, sa mise en page révèle son organisation, le choix de son iconographie traduit un certain fonctionnement mental » (Lombard, 2001 : 109-118). Toute communication est orientée, elle vise une certaine fin ainsi que le confirme ( Jakobson, $1963: 211$ ) lorsqu'il dit « ...toute communication est orientée vers un but, mais les objectifs varient ".

19 En temps de conflit ou de crise, la communication s'intensifie pour devenir quasiment omniprésente. À ce stade, elle dessert plutôt l'objectif premier qui est de maintenir la population dans un état de calme et d'éviter la panique. Si l'on communique, ce n'est pas pour rien et tout dépend du camp dans lequel on se situe et de l'objectif visé. Pour les terroristes par exemple, le but est de semer la panique, créer le choc, appâter de 
futures recrues. Ils utilisent pour cela les réseaux sociaux en montrant des images censées faire naître un désir d'aventure chez les candidats au djihad. L'attentat luimême est un moyen de communication. Au-delà du choc c'est la médiation de la violence qui instaure un climat de terreur. La vidéo de l'otage français Hervé Gourdel, dont la décapitation est filmée et diffusée massivement sur les réseaux sociaux en est l'illustration (Schneiderman, 2003).

\section{Prise de décision en temps de crise}

$\mathrm{Au}$ milieu de toutes ces problématiques, il faut tenir compte de la personne dans son entité qui doit prendre une décision efficace. Or dans un contexte de conflit, la diversité de sources d'information et les orientations des uns et des autres, il est difficile pour une personne de déterminer avec efficacité les indicateurs nécessaires qui conduiront à sa prise de décision. Il devient crucial pour lui d'adopter une méthodologie qui guidera sa recherche d'information. Cette méthodologie peut être inspirée du concept de l'Intelligence Économique (IE) qui de par sa définition, aide à cadrer la collecte, le traitement et la gestion de l'information. Nous pensons que ce n'est pas la diversité de sources d'information qui pose problème, ni les pratiques des médias, mais l'enjeu se situe au niveau du processus décisionnel. Le défi pour celui qui doit décider est d'en prendre conscience et d'avoir une capacité d'adaptation selon le problème auquel il fait face.

\section{Qu'est-ce que l'intelligence économique?}

Certains auteurs définissent l'Intelligence Économique (IE) comme un

« ensemble d'actions coordonnées de recherche, de traitement et de diffusion de l'information utile aux acteurs économiques en vue de son exploitation à des fins stratégiques et opérationnelles. Ces diverses actions sont menées légalement avec toutes les garanties de protection nécessaires à la préservation du patrimoine de l'entreprise, dans les meilleures conditions de qualité, de délais et de coût » (Martre, $1994: 12$ ).

Pour Revelli (1998: 15), c'est « un processus de collecte, de traitement et de diffusion de l'information qui a pour objet la réduction de la part d'incertitude dans la prise de toute décision stratégique ».

En tant que processus, nous pouvons décomposer l'IE en plusieurs étapes afin d'atteindre un objectif qui est la prise de décision. De sa définition, Ndjock (2016) ressort quatre mots clés sur lesquels il est important d'insister :

- le processus qui signifie que l'IE n'est pas une action, mais un ensemble d'actions coordonnées ;

- l'information qui est la matière première, l'objet de recherche du processus ;

- la part d'incertitude: sachant que toute prise de décision est une prise de risque, la définition de l'IE permet de réduire la part d'incertitude liée à la prise de ce risque ;

- la décision qui est la cause du déclenchement du processus, le but pour lequel le processus est engagé.

Rapportant cette définition à notre sujet, nous voyons que prendre une décision n'est pas un acte isolé, mais plutôt un processus qui se situe dans le temps et peut-être bien dans l'espace. En effet, une décision correspond à un environnement, un contexte précis. En temps de guerre ou de conflit, il faut agir vite, mais pas forcément 
précipitamment. La cause du déclenchement du processus décisionnel est la décision et c'est l'utilisation finale de l'information qui conduit à la recherche d'information. La décision prise peut servir de base de réflexion par temps de paix, car nous estimons que le processus n'est pas linéaire et ses étapes sont récursives.

\section{Le processus d'intelligence économique et la prise de décision}

\section{Le processus décisionnel}


Choix, il faut choisir la meilleure solution parmi différentes alternatives ; très souvent, le décideur s'appuie sur des faits concrets pour prendre sa décision, mais plus risqué, il peut se baser sur son intuition. La logique voudrait qu'on reste dans la rationalité pour décider ; malheureusement comme c'est généralement le cas en temps de crise, il est rarement fait appel à cette notion. Les médias sont la source principale d'information et l'on ne dispose pas de toute l'information nécessaire. D'ailleurs, Simon remet en question cette rationalité du décideur. Il estime que l'on suppose souvent que le décideur dispose d'informations complètes et connaît à l'avance les conséquences de ses décisions, mais ce n'est pas le cas, car en pratique, en pratique, il est très difficile, d'obtenir des informations complètes. Ce qui veut dire que la rationalité du décideur est limitée. Par conséquent, au moment de la prise de décision, il est quasiment impossible de prouver que la décision prise est la meilleure! Le décideur s'arrêtera donc à une décision lui paraissant satisfaisante.

\section{L'Intelligence économique}

L'IE apporte une démarche permettant de « minimiser la part d'incertitude » dans la prise de décision. Nous appuyant sur les travaux d'Herbert Simon (1957:241-260), nous nous positionnons au niveau de la stratégie «du regret maximum le plus faible». Le raisonnement est le suivant :

- Supposons que l'événement E1 se réalise, la meilleure stratégie est alors S1. Si la stratégie effectivement choisie est $\mathrm{S} 1$, l'entreprise n'a pas de regrets d'où 0 dans la case S1/E1.

- Si par contre la stratégie effectivement choisie est S2, on réalise un gain de 50 alors qu'avec S1 ce gain aurait été de 100 . Le regret s'exprime par la différence $100-50=50$ d'où 50 dans la case $\mathrm{E} 1 / 2$, et ainsi de suite ...

On relève ensuite les regrets maximums pour chaque stratégie :

- 350 pour la stratégie 1

- 250 pour la stratégie 2

- 150 pour la stratégie 3

- 200 pour la stratégie 4

On choisit ensuite la stratégie pour laquelle le regret maximum est le plus faible soit la stratégie 3.

David \& Thierry (2003) considèrent l'IE comme l'utilisation de l'information pour le processus décisionnel stratégique. Les résultats des recherches en Management et en Psychologie de (Falque \& Bougon, 2009) ont révélé quatre modèles de décision à savoir :

- l'approche rationnelle;

- l'approche de la poubelle ;

- approche politique ;

- choix satisfaisant.

Dans le cadre de cette étude, les approches rationnelles et du choix satisfaisant sont privilégiées puisqu'il est indispensable que l'information soit exempte de toute contrainte pour aider à prendre les décisions adéquates. Afin de veiller à ce que les décisions soient de plus en plus fondées sur des informations correctes, il y a lieu d'appliquer une méthodologie rigoureuse et efficace dans le processus. Cette méthodologie découle du concept d'Intelligence économique (IE) qui de par sa définition, aide à cadrer la collecte, le traitement et la gestion de l'information. Ses étapes clairement définies du début à la fin du processus décisionnel guident tant 
l'utilisateur dans la collecte d'information que le décideur dans la prise de décision s'appuyant essentiellement sur les travaux de David (2005) au sein de l'équipe SITELORIA qui ressortent que dans la procédure traditionnelle de résolution d'un problème décisionnel, lorsqu'un problème est identifié, la procédure a consisté jusqu'à présent à rechercher (collecter) des informations afin de lui trouver une solution comme le confirme l'illustration suivante :

Figure 1 : Procédure traditionnelle de résolution d'un problème décisionnel - CVI

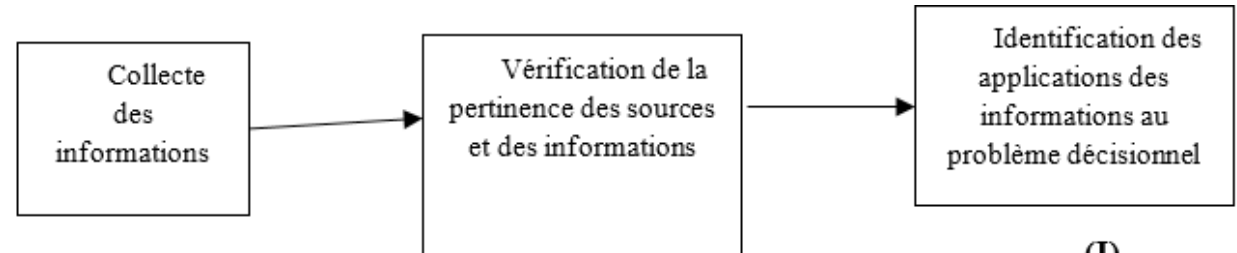

(C)

(V)

(I) de la figure 1 qui devient : Figure 2 : Approche améliorée de résolution d'un problème décisionnel - IVC

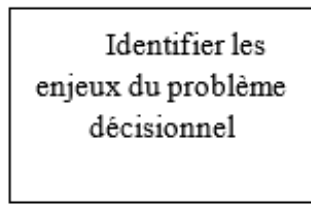

(I)

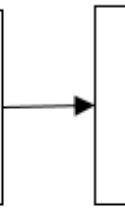

Il est prouvé que toute activité de recherche d'informations reste stérile si le contexte d'utilisation de ces informations n'est pas bien spécifié avant de commencer la recherche ce d'autant plus que c'est l'utilisation potentielle qui oriente la recherche et non l'inverse. Collecter les informations avant l'identification de ses applications éventuelles est une activité infructueuse.

- identification d'un problème décisionnel ;

- traduction du problème décisionnel en un problème de recherche d'information ;

- identification des sources pertinentes d'information;

- collecte des informations pertinentes ;

- analyse des informations collectées pour extraire des indicateurs pour la décision ;

f) interprétation des indicateurs;g) prise de décision.

Il propose ensuite, qu'au lieu de vérifier les sources d'informations, leurs pertinences et leurs utilisations potentielles après les avoir collectées, de :

- commencer par définir le problème décisionnel aussi explicitement possible ;

- traduire la représentation du problème décisionnel en problème de recherche d'informations ;

- identifier les sources les plus pertinentes de l'information.

De cette procédure ressort une nouvelle approche par l'inversion des étapes du schéma

David (2005) que nous avons déjà cité affirme qu'il est «indispensable de bien comprendre le problème décisionnel qui se pose afin de pouvoir déterminer la 
pertinence des informations qui seront nécessaires pour sa résolution, car, au cœur du problème d'IE se trouve donc la question de la pertinence de l'information » (David, $2005: 4)$.

En quoi la méthodologie énoncée par l'IE permet-elle de «réduire la part d'incertitude » dans la prise de décision? Si nous appliquons l'approche améliorée de résolution d'un problème décisionnel nous obtenons :

Identification des enjeux du problème décisionnel. Il est préconisé de définir aussi clairement que possible le problème décisionnel. Pour notre père de famille, le problème est la sécurité des siens. Il faut se mettre à l'abri et ne pas prendre de risques. Ce n'est pas parce que les informations défilent en boucle que des bombes humaines explosent dans les lieux publics qu'il faut tout de suite prendre la décision de rester chez soi. L'IE voudrait que tous les paramètres soient étudiés par exemple la situation géographique par rapport aux explosions. Pour prendre le cas du Nord-Cameroun, le père de famille à Yaoundé risque de se barricader chez lui et se faire prisonnier simplement en regardant la télévision, surfe sur internet ou lit les posts sur les réseaux sociaux parce qu'il ne lit et ne suit que " Une bombe a explosé ce matin au Cameroun ». C'est le cas par exemple lors des explosions au Bataclan en France. De nombreuses familles en Afrique ont appelé leurs proches en France pour avoir des nouvelles sans prendre en considération le lieu géographique du drame, ou au Nigéria lors de l'explosion au siège de l'ONU à Abuja. Certains passagers en partance pour Lagos ont annulé leur voyage croyant que c'est tout le Nigéria qui est concerné. Plus récemment avec l'accident ferroviaire dans la ville d'Éséka au Cameroun. Les amis et proches se sont rués sur les moyens de communication pour prendre des nouvelles des familles des villes et villages qui portant loin du lieu du drame. Dans cette situation, ce ne sont pas l'ampleur des explosions qui déclenche la psychose, mais bien entendu l'information véhiculée. Dans ces deux cas, les explosions n'ont pas été entendues en Afrique (pour le cas du Bataclan) ou en Europe (pour l'ambassade, l'accident ferroviaire), mais bien ce que les proches ont vu à la télé, suivi à la radio ou lu sur les réseaux sociaux qui déclenche la psychose.

Vérification (identification) des sources et informations pertinentes : il faut identifier les différentes sources d'information (la télévision, les réseaux sociaux, les blogs, le téléphone, la famille, ...). Que disent les médias? Leur diversité est en même temps un avantage et une source de dispersion si l'on ne prête pas attention. La pertinence de l'information dans ce cas se détermine à la capacité qu'a une information de faciliter la résolution du problème décisionnel. Il est de même de la source d'information. Elle sera pertinente si elle donne la possibilité de répondre clairement aux questions posées.

Collecte d'informations. C'est l'utilisation potentielle de l'information qui oriente sa recherche et non l'inverse. Pour optimiser sa recherche et minimiser la part d'incertitude dans la prise de décision, il faut savoir pour quelle raison a-t-on besoin de telle ou telle information? Quelle en sera son utilisation? Ce n'est qu'après avoir répondu à cette question que la recherche d'informations peut être fructueuse. Ce que confirme David quand il précise que "C'est la connaissance sur l'utilisation finale de l'information qui permet de donner un jugement sur sa pertinence » (David, 2008 : 209-231). 


\section{Conclusion}

Les enjeux de la communication de l'information en temps de crise dus à la diversité des sources d'information sont pour la plupart essentiellement liés aux enjeux du processus décisionnel, car il est question à la fin de prendre une décision. Quel que soit le niveau hiérarchique donné (à l'échelle de l'être humain ou en entreprise), le processus décisionnel en temps de crise ou de conflit reste le même. La prolifération et la diversité des sources d'information sont un avantage, mais aussi un inconvénient dans le processus décisionnel cependant, la méthodologie proposée par l'Intelligence économique permet au décideur de minimiser la part d'incertitude dans la prise de décision. Il arrive que des événements mineurs et des actions individuelles ponctuelles exercent une influence imprévisible sous l'effet des médias. La tâche du décideur se trouve ainsi compliquée, car il ne peut prévoir les effets des aléas. Au-delà des différentes conséquences induites par les médias lors de la gestion de la crise, il est essentiel d'adopter une méthodologie qui permette de prendre de décision efficace avec des marges d'erreur réduites. Chacun a accès aux mêmes informations (terroristes, personnels, gouvernement), il ne faut surtout pas perdre de vue le fait que la diffusion d'une information n'est jamais neutre, le décideur doit prendre conscience de tous ces paramètres et ne pas se laisser submerger au risque de prendre de décisions partant sur des bases erronées.

En outre, le traitement médiatique de l'information est amené à évoluer avec le développement d'internet et la multiplication de la diversité des sources ce qui modifie aussi l'accès à l'information. Garder un esprit critique, prendre du recul et accorder à chaque information sa valeur et la recherche de l'information sera plus rationnel, plus réfléchi et plus sélectif. En laissant des vides organisationnels dus aux routines qui ne peuvent plus avoir cours dans la recherche d'information, la diversité des sources d'information crée des conditions nouvelles et ouvre de nouveaux champs à des potentialités de recherche et d'innovation plus grandes. Elle modifie l'organisation et les schémas des décideurs et permet d'explorer de nouvelles approches méthodologiques dans le processus décisionnel.

\section{BIBLIOGRAPHIE}

Saliou Akin, La Communication politique : utilités et difficultés, Edilivre, Paris, 2008.

Salomon Asch, Essays in cognition and social psychology, Macmillan, New-York, 1990.

Guillaume Barbalat, (2007) « Le processus de prise de décision chez le sujet addict », Psychotropes, volume 13 (2), 2007, pp. 91-105.

Jean Cazeneuve, «Théories de la communication », Les cahiers de la publicité, volume 5 (1), 1963, pp. 11-17.

Hervé Courtot, «Communication des risques dans les projets », Communication et organisation, 2001, http://communicationorganisation.revues.org/2553, consulté le 9 mars 2016. 
Xavier Couture, La dictature de l'émotion. Où va la télévision ? Editions Audibert, Paris, 2005.

Amos David, «L'intelligence économique et les systèmes d'informations : problématiques et approches de solutions » Equipe SITE-LORIA. Université de Nancy2. Veille stratégique : Mise en œuvre et valorisation de la veille stratégique en entreprise, Jun 2005, Alger, Algérie, 2005, https://hal.inria.fr/inria-00000255, consulté le 3 mars 2016.

Amos David, «L'information pertinente en intelligence économique », Fabrice Papy (dir.)., Problématiques émergeantes dans les sciences de l'information, Paris. Hermès, Paris, 2008, pp. 209- 231.

Odile Thierry \& Amos David, « L'architecture EQuA2te et son application à l'Intelligence Economique », ISDM : Informations, Savoirs, Décisions et Médiations, (8)2003, https://hal.inria.fr/ inria-00099690/en, consulté le 15 mars 2016.

Larent Falque, Bernanrd Bougon, Pratiques de la décision, développer ses capacités de discernement, Dunod, Paris, 2009.

Jean-Pierre Goor, « Le rapport sur le sentiment d'insécurité et l'influence des médias », Charleroi : Actes de la journée d'études. Forum Belge pour la Prévention et la Sécurité Urbaine ASBL, (dir.) V. Ketelaer et L. Nolet, 2008, pp. 9-16.

Guibert Houllier, « Les politiques de communication rennaises pour un positionnement européen », Communication et organisation, (35), 2009, pp. 97-110.

Roman Jakobson, Essai de linguistique générale, Tome 1 « Les fondements du langage », Editions de Minuits, Paris, 1963.

Michel Joly \& Jean-Louis Muller, « De la gestion de projet au management par projet », Afnor, (45), 1994, pp. 123-134.

Thierry Libaert, « La communication sensible, nouvelle discipline de communication organisationnelle », Magazine de la communication de crise et sensible, 2011, pp. 9-15.

François Lombard, « Quand la communication prend le dessus sur la technique », Revue de l'EPI, (104), 2001, pp. 109-118.

Henri Martre, Intelligence économique et stratégie des entreprises, La Documentation française. Paris, 1994.

Robert Michit, « Résolution de problèmes et prise de décision », Communication et organisation, (14), 1998, mis en ligne le 26 mars 2012URL : http://communicationorganisation.revues.org/2137, consulté le 19 avril 2016.

Fleur Nadine Ndjock, « Observatoire dynamique comme outil d'aide à la décision appliqué à un système éducatif : cas du Cameroun - Approche de l'intelligence économique », Thèse de doctorat en cours au Cnam, Paris, 2016.

Carlos Revelli, Intelligence stratégique sur Internet, Dunod, Paris, 1998. Daniel Schneiderman, Le Cauchemar médiatique, Denoël, Paris, 2003.

Herbet Simon, "A behavioural model of rational choice", in H.A. Simon, Editor, Models of man : social and rational ; mathematical essays on rational human behavior in a social setting, J. Wiley, New York, 1957, pp. 241--260.

Edward Thorndike, The Psychology of arithmetic, New-York : Macmillan, 1922. 


\section{NOTES}

1. Sentiment d'insécurité et impact des médias - Actes de la journée d'études - Charleroi, 25 juin 2008.

2. Idem.

\section{RÉSUMÉS}

Cet article a pour objectif de montrer sur la base d'un retour d'expérience issu des travaux effectués dans le cadre d'un projet de thèse, que les enjeux de la communication de l'information en temps de crise dus à la diversité des sources d'information sont pour la plupart essentiellement liées aux enjeux du processus décisionnel. Nous pensons qu'une communication est faite en temps de crise pour informer, pour permettre de prendre des dispositions (décider) s'il y a lieu et c'est ce dernier aspect qui nous intéresse : quel impact la diversification des sources d'information a-t-elle sur le processus décisionnel en temps de crise? «La prise de décision est un processus cognitif complexe visant à la sélection d'un type d'action parmi différentes alternatives » et nous savons qu'en temps normal et particulièrement en période de crise que prendre une décision est un acte crucial. La décision doit être rapide et efficace. Pour cette raison, le décideur doit disposer de l'information pertinente. Or, il se trouve que plusieurs facteurs influencent la communication de l'information en temps de crise ou de conflit. Ces facteurs sont déterminants dans la demande et le traitement de l'information et jouent un rôle primordial dans la prise de décision finale. L'environnement conflictuel, la diversité des sources d'information, le problème de crédibilité des médias rendent particulièrement ardue, la détermination des indicateurs, la collecte et le traitement de l'information. Or, il est primordial que dans un tel environnement, l'information soit exempte de toute contrainte pour aider à prendre les décisions adéquates. Le défi pour celui qui doit décider est d'en prendre conscience et d'avoir une capacité d'adaptation selon le problème auquel il fait face. Nous pensons que pour y arriver, il convient d'appliquer une méthodologie rigoureuse et efficace dans l'ensemble du processus qui commence dès l'accès à l'information jusqu'à la prise de décision. Cette méthodologie peut être inspirée du concept de l'Intelligence Économique (IE) qui de par sa définition, aide à cadrer la collecte, le traitement et la gestion de l'information. Ses étapes clairement définies du début à la fin du processus décisionnel guident tant l'utilisateur dans la collecte d'information que le décideur dans la prise de décision.

This paper aims to show on the basis of feedback from the work carried out as part of a thesis project, the challenges of communicating information in times of crisis due to the diversity of information sources are mostly related primarily to issues of decision making. We believe that communication is made in times of crisis to inform, to help make arrangements (decision) if necessary and it is this aspect that interests us: what impact the diversification of sources of information has on decision making? "Decision making is a complex cognitive process of selecting a type of action among alternatives" and we know that in normal times and especially in times of crisis, making a decision is a crucial act. The decision must be fast and efficient. For this reason, the decision maker must have the relevant information. Now it happens that several factors influence the communication of information in times of crisis or conflict. These factors are crucial in the application and processing of information and play a key role in the final 
decision. The conflict environment, the diversity of information sources, media credibility problem make it particularly difficult, determination of indicators, collection and processing of information. However, it is important that in such an environment, the information is free from coercion to help make the right decisions. The challenge for the one who has to decide is to be aware and have a resilience depending on the problem it faces. We believe that to happen, it is important to apply a rigorous and effective methodology in the whole process that begins access to information to decision-making. This methodology can be inspired by the concept of Competitive Intelligence ( $\mathrm{CI}$ ) which, according to its definition, helps to frame the collection, processing and information management. It clearly defined the steps from the beginning to the end of the decision-making process as guide the user in gathering information that the decision maker in the decision making.

Dieser Artikel zielt darauf ab, auf der Grundlage der Rückmeldungen aus den durchgeführten Arbeiten im Rahmen einer Diplomarbeit zu zeigen, die Herausforderungen der Informationen in Krisenzeit in Verbindung steht aufgrund der Vielfalt der Informationsquellen sind meist in erster Linie auf Fragen der Entscheidungsfindung im Zusammenhang. Wir glauben, dass die Kommunikation in Zeit der Krise gemacht $\mathrm{zu}$ informieren, $\mathrm{zu}$ helfen, Vereinbarungen (entscheiden) zu machen ist notwendig, und es ist dieser letzte Aspekt, der uns interessiert: welche Auswirkung hat die Diversifizierung der Informationsquellen in Zeit der Krise auf um zu entscheiden?

„Die Entscheidung nehmen ist ein komplexes kognitives Verfahren,die eine Art von Aktion zwischen Alternativen wählen abzielt" und wir wissen, dass in normalen Zeit und vor allem in Krisenzeit eine Entscheidung zu treffen ist ein wichtiger Akt. Die Entscheidung muss schnell und effizient sein. Aus diesem Grund muss der Entscheider die relevanten Informationen haben. Nun kommt es vor, dass mehrere Faktoren beeinflussen die Übermittlung von Informationen in Zeit der Krise oder einem Konflikt. Diese Faktoren sind von entscheidender Bedeutung in der Anwendung und die Verarbeitung von Informationen und spielen eine wichtige Rolle bei der endgültigen Entscheidung. Die Konfliktuelumwelt, die Vielfalt der Informationsquellen, Medien Glaubwürdigkeitsproblem macht es besonders schwierig, zu identifizieren Indikatoren, die Erfassung und Verarbeitung von Informationen. Allerdings ist es wichtig, dass eine Umgebung, in solchen, die Informationen frei von Zwang, um richtigen Entscheidungen zu machen helfen. Die Herausforderung für den, der zu entscheiden hat, ist sich dessen bewusst zu sein und eine Elastizität haben das Problem, je sie konfrontiert ist. Wir glauben, dass das geschehen kann, sollte eine strenge und wirksame Methodik in den gesamten Prozess anwenden, die den Zugang zu Informationen über die Entscheidungsfindung beginnt. Diese Methodik kann durch das Konzept der wirschaftliche Klugheit (WK), die durch ihre Definition inspiriert werden, um die Erfassung, Verarbeitung und Verwaltung von Informationen helfen umrahmen. Seine klar definierten Stufen von Beginn des Entscheidungsprozesses zu beenden, wie der Benutzer in dem Sammeln von Informationen, dass die Entscheidungsträger in der Entscheidungsfindung.

\section{INDEX}

Schlüsselwörter : Information, Kommunikation, Krise, wirtschaftliche Intelligenz, Entscheidungsfindung, konfliktuelles Umfeld

Keywords : information, communication, crisis, competitive intelligence, decision making, conflict environment

Mots-clés : information, communication, crise, intelligence économique, processus décisionnel, environnement conflictuel 
AUTEUR

FLEUR NADINE NDJOCK

Conservatoire National des Arts et Métiers (CNAM) 